\title{
Cooperative Spectrum Sensing and Localization in Cognitive Radio Systems Using Compressed Sensing
}

\author{
Wael Guibène and Dirk Slock \\ Mobile Communications Department, EURECOM, SophiaTech Campus, 450 Route des Chappes, 06410 Biot, France \\ Correspondence should be addressed to Wael Guibène; guibene@eurecom.fr
}

Received 18 April 2013; Revised 20 June 2013; Accepted 26 July 2013

Academic Editor: Luca Reggiani

Copyright ( 2013 W. Guibène and D. Slock. This is an open access article distributed under the Creative Commons Attribution License, which permits unrestricted use, distribution, and reproduction in any medium, provided the original work is properly cited.

\begin{abstract}
We propose to fuse two main enabling features in cognitive radio systems (CRS): spectrum sensing and location awareness in a single compressed sensing based formalism. In this way, we exploit sparse characteristics of primary units to be detected, both in terms of spectrum used and location occupied. The compressed sensing approach also allows to overcome hardware limitations, in terms of the incapacity to acquire measurements and signals at the Nyquist rate when the spectrum to be scanned is large. Simulation results for realistic network topologies and different compressed sensing reconstruction algorithms testify to the performance and the feasibility of the proposed technique to enable in a single formalism the two main features of cognitive sensor networks.
\end{abstract}

\section{Introduction}

Cognitive radio (CR) is a smart wireless communication concept that is able to promote the efficiency of the spectrum usage by exploiting the free frequency bands in the spectrum, namely, spectrum holes $[1,2]$.

Detection of spectrum holes, namely, spectrum sensing, is the first step towards implementing a cognitive radio system.

The major problem for spectrum sensing arises in wideband radio, when the radio is not able to acquire signals at the Nyquist sampling rate due to the current limitations in Analog-to-Digital Converter (ADC) technology [3]. Compressive sensing makes it possible to reconstruct a sparse signal by taking less samples than Nyquist sampling, and thus wideband spectrum sensing is doable by compressed sensing (CS). A sparse signal or a compressible signal is a signal that is essentially dependent on a number of degrees of freedom which is smaller than the dimension of the signal sampled at Nyquist rate. In general, signals of practical interest may be only nearly sparse [3]. And typically signals in open networks are sparse in the frequency domain since depending on location, and at some times the percentage of spectrum occupancy is low due to the idle radios $[1,4]$.
In CS, a signal with a sparse representation in some basis can be recovered from a small set of nonadaptive linear measurements [5]. A sensing matrix takes few measurements of the signal, and the original signal can be reconstructed from the incomplete and contaminated observations accurately and sometimes exactly by solving a simple convex optimization problem $[3,6]$. In $[7,8]$, conditions on this sensing matrix are introduced which are sufficient in order to recover the original signal stably. And remarkably, a random matrix fulfills the conditions with high probability and performs an effective sensing $[5,9]$.

Another step towards the feasibility and a real implementation of cognitive radio systems is the problem of location awareness $[10,11]$. This problem arises when we do consider a realistic scenario in hybrid overlay/underlay systems [12,13], when these spectrum opportunities permit cognitive radios to transmit below the primary users tolerance threshold. In this case, the cognitive radio (CR) has to estimate robustly the primary users locations in the network in order to adjust its transmission power function of the estimated location in the network. The knowledge of position information in CR system (CRS) is also an enabler of location-based beamforming as shown in [14] and also as shown in the ICTWHERE2 project, a whole framework of location-aided 
PHY/MAC layer design for advanced cognitive radios [15] with novel concepts of spectrum sensing techniques based on location information [16], to multicell multiuser MIMO systems with location-based CSIT [17].

In our approach (part of the work presented in this paper was accepted and presented in WIMOB 2012 [10], 8th IEEE International Conference on Wireless and Mobile Computing, Networking and Communications, and CAMAD 2012 [11], IEEE 17th International Workshop on Computer Aided Modeling and Design of Communication Links and Networks), we propose to analyze all these arisen problems. During the problem formulation and when analyzing more deeply the equations related to each question apart, we will make the link between the formulation of spectrum sensing, location awareness, and the hardware limitation by describing those problems in a unique compressed sensing formalism.

The paper is organized as follows. In Section 2, we start by giving a first overview on the spectrum sensing techniques. Section 3 is dedicated to introducing the compressed sensing framework we are targeting during the problem formulation. Section 4 is exposing the remonstration techniques we are using to overcome the sparsity problem. In Section 5, we introduce the system model of the considered CR system, and in Section 6 we make the link between spectrum sensing and localization in a common CS formalism. Finally, in Section 7 we give the simulations results for realistic CR configuration, and in Section 8 we conclude on the effectiveness of the proposed CS approach for sensing/localization in CR systems.

\section{Related Work}

As previously stated, cognitive radio (CR) is presented [18] as a promising technology in order to handle this shortage and misuse of spectral resources. According to FCC, a radio is considered cognitive when it has the following capabilities.

(i) Frequency agility: the ability of a radio to change its operating frequency to optimize use under certain conditions.

(ii) Dynamic frequency selection: the ability to sense signals from other nearby transmitters in an effort to choose an optimum operating environment.

(iii) Location awareness: the ability for a device to determine its location and the location of other transmitters; that would help selecting the appropriate operating parameters such as the power and frequency allowed at the given location.

(iv) Negotiated use: incorporate a mechanism that would enable sharing of spectrum under the terms of a prearranged agreement between a licensed (primary) and nonlicensed (secondary) users.

(v) Adaptive modulation: the ability to modify and adapt transmission characteristics and waveforms to exploit opportunities to use spectrum.

(vi) Transmit power control: allows transmission at full power limits when necessary.
The presented work fits in the context of spectrum sensing/localization framework of cognitive radio systems (CRS) and more precisely cooperative transmitter detection/localization. In this context, many statistical approaches for spectrum sensing have been developed. The most performing one is the cyclostationary features detection technique $[19,20]$. The main advantage of the cyclostationarity detection is that it can distinguish between noise signal and PU transmitted data. Indeed, noise has no spectral correlation, whereas the modulated signals are usually cyclostationary with nonnull spectral correlation due to the embedded redundancy in the transmitted signal. The cyclostationary features detector is thus able to distinguish between noise and PU.

The reference sensing method is the energy detector (ED) [19], as it is the easiest to implement. Although the ED can be implemented without any need for a priori knowledge of the PU signal, some difficulties still remain for implementation. First of all, the only primary user (PU) signal that can be detected is the one having an energy above some given threshold. So, the threshold selection in itself can be problematic as the threshold highly depends on the changing noise level and the interference level. Another challenging issue is that the ED approach cannot distinguish the PU from the other secondary user (SU) sharing the same channel. Cyclostationary features detector (CFD) is more robust to noise uncertainty than an ED. Furthermore, it can work with lower SNR than ED.

More recently, a detector based on the signal space dimension based on the estimation of the number of the covariance matrix independent eigenvalues has been developed [21]. It was presented that one can conclude on the nature of this signal based on the number of the independent eigenvectors of the observed signal covariance matrix. The Akaike information criterion (AIC) was chosen in order to sense the signal presence over the spectrum bandwidth. By analyzing the number of significant eigenvalues minimizing the AIC criterion, one is able to conclude on the nature of the sensed subband. Specifically, it is shown that the number of significant eigenvalues is related to the presence or not of data in the signal.

Still in these state-of-the-art techniques, a key assumption of perfect signals acquisition is always made. But real world signals and systems are intrinsically sparse, as sparsity may come from different factors. A first one, which is considered in this paper, is the inability of the ADC to acquire signals at a Nyquist rate resulting in inaccurate and incomplete data. A second source of sparsity may be that spectrum use in open wireless networks is sparse, that is to say, few resources are used, and in most of geographic areas and/or period of time, resources are left idle.

\section{Compressed Sensing Framework}

In this section, we are considering sparse signals/vectors reconstruction.

A given $d$-dimensional vector is assumed to be $s$-sparse if it has $s$ or fewer nonzero coordinates, that is to say;

$$
x \in \mathbb{R}^{d}, \quad\|x\|_{0} \triangleq|\operatorname{supp}(x)| \leq s \ll d,
$$


where we denote by $\|\cdot\|_{0}$ the quasinorm, and for $1 \leq p<\infty$, $\|\cdot\|_{p} \triangleq\left(\sum_{i=1}^{d}\left|x_{i}\right|^{p}\right)^{1 / p}$ is the usual $p$-norm. In real world, we will not encounter perfect sparse signals, but signals whose coordinates satisfy a power law decay, that is to say $x$ satisfy the following equation:

$$
\left|x_{i}^{*}\right| \leq R i^{(-1 / q)},
$$

where $x^{*}$ is a nonincreasingly rearranged version of $x, R$ is some positive constant, and $q$ is satisfying $0<q<1$. Sparse vectors recovery algorithms tend to reconstruct sparse vectors from a small set of measurements. Each of these measurements can be viewed as an inner product between a given vector, say $\phi_{i} \in \mathbb{R}^{d}$, and the vector $x \in \mathbb{R}^{d}$. Collecting the $m$ measurement in a single matrix, we thus build an $m \times d$ measurement matrix, say $\boldsymbol{\Phi}=\left[\begin{array}{lll}\phi_{1} & \cdots & \phi_{m}\end{array}\right]$.

Theoretically speaking, recovering $x$ from its measurement $u=\Phi x \in \mathbb{R}^{m}$ is equivalent to solving the $l_{0}$-minimization problem as follows:

$$
\min _{z \in \mathbb{R}^{d}}\|z\|_{0} \text { subject to } \Phi z=u \text {. }
$$

If $x$ is $s$-sparse and $\Phi$ is one to one (a matrix is told to be one to one if it is representing an injective transformation from one space to another), on all $2 s$-sparse vectors, then the solution of (3) must be the signal $x$. Indeed, say $z$ is a solution and given the fact that $x$ is an obvious solution, then $z-x$ must be a kernel of $\boldsymbol{\Phi}$. But $z-x$ is a $2 s$-sparse vector and given the assumption that $\Phi$ is one to one, then $z=x$. Thus, theoretically speaking the $l_{0}$-minimization is a perfect solution to the reconstruction problem. Unfortunately it is shown in the literature [22] that the $l_{0}$-minimization is an NPhard problem and numerically unfeasible.

This problem can be overcome by means of compressed sensing (CS). A first approach is to use basis pursuit (BP) algorithm in order to relax the $l_{0}$-minimization to an $l_{1}$ minimization formalism. BP requires stronger hypothesis on $\Phi$, so it does not have only to verify injection on sparse vectors property, but it has been shown that the relationship between $m, d$, and $s$ is given by $m=s \log O(1) d . l_{1}$-minimization often relies on linear programming, and since there is no linear bound for such techniques, BP approaches are quite slowly convergent techniques. The second approach is to use greedy algorithms such as orthogonal matching pursuit (OMP) [23], stagewise orthogonal matching pursuit (StOMP) [24], or iterative thresholding $[25,26]$. Those approaches are based on the iterative computation of the signal's support. As for BP, $m, d$, and $s$ are linked parameters such as $m=$ $O(s \log d)$. Once the support $S$ of the signal is computed, $x$ is reconstructed from the measurement vector $u$ as $x=\left(\boldsymbol{\Phi}_{S}\right)^{\dagger} u$, where $\boldsymbol{\Phi}_{S}$ is the restriction of $\boldsymbol{\Phi}$ to the columns indexed by $S$, and $\dagger$ is the pseudoinverse operator (recalling that for a given matrix $\left.A, A^{\dagger}=\left(A^{*} A\right)^{-1} A^{*}\right)$. The main advantage of greedy approaches is their convergence time, as they are faster than BP, but they lose in stability compared to BP. Another class of CS algorithms recently emerged in order to shorten the gap between greedy algorithms and BP. From these algorithms, we may cite regularized orthogonal matching pursuit
(ROMP) [27] and compressive sampling matching pursuit (CoSaMP) [28]. These two algorithms provide a similar guarantee of stability as BP with the same iterative property of fast convergence as greedy algorithms.

\section{Reconstruction Algorithms}

In our work, we will consider one algorithm per class to be studied for spectrum sensing and localization purposes. We will thus introduce first of all BP, then OMP, and finally CoSaMP that will be used afterwards for the target applications.

4.1. Basis Pursuit. Since the problem as formulated in (3) is an NP-hard problem and numerically unfeasible, let us introduce a first approach to solve this problem. One may consider first a mean square approach to solve the problem as follows:

$$
\min _{z \in \mathbb{R}^{d}}\|z\|_{2} \text { subject to } \Phi z=u \text {. }
$$

Since the minimizer, say $x^{*}$, must satisfy $\Phi x^{*}=u=\Phi x, x^{*}$ must be in the subspace $K=x+\operatorname{ker} \Phi$. Actually, $x^{*}$ as defined in (4) is the exact contact point between the smallest Euclidian ball centered at the origin and the subspace $K$. As shown in Figure 1, in the mean square approach there is no need to have $x^{*}$ coinciding with the actual signal $x$. This is due to the fact that Euclidian geometry ball is not a good detector of sparsity.

In this case, in order to solve (3), we may opt for an $l_{1}$ approach. In this case, $x^{*}$ would meet the contact point between the $l_{1}$ ball (also named octahedron) centered at the origin and the subspace $K$.

A first idea could be relaxing the problem into an $l_{1}$ minimization:

$$
\min _{z \in \mathbb{R}^{d}}\|z\|_{1} \text { subject to } \Phi z=u .
$$

Authors in [7] have proved that for measurement matrices satisfying a certain quantitative property called restricted isometry property, $l_{0}$ and $l_{1}$ become equivalent.

4.2. Orthogonal Matching Pursuit. OMP is based on subgaussian measurement matrices to reconstruct sparse signals. If $\Phi$ is verifying such condition (subgaussian property), then $\Phi^{*} \Phi$ is close to identity. In this case, a nonzero coordinate of $x$ would maximize the observation $y=\boldsymbol{\Phi}^{*} \boldsymbol{\Phi} x$, and that is how we iteratively reconstruct the support of $x$. OMP is shown to be fast but not as stable as BP.

Algorithm 1 gives the pseudocode for OMP implementation.

Once the support $I$ of the signal $x$ is found, the estimate $\hat{x}$ can be reconstructed as $\widehat{x}=\Phi_{I}^{\dagger} u$. The algorithm simplicity allows a fast reconstruction as it iterates $s$ times, and over each iteration, it selects one among $d$ elements in $O(d)$ time and multiplies by $\Phi^{*}$ in an $O(m d)$ time period and finally solves a least squares problem in $O\left(s^{2} d\right)$. So the cost of such technique is $O(s m d)$ operations. 


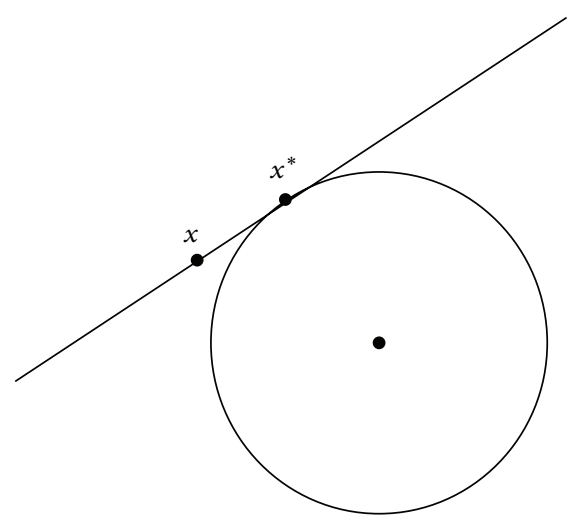

(a)

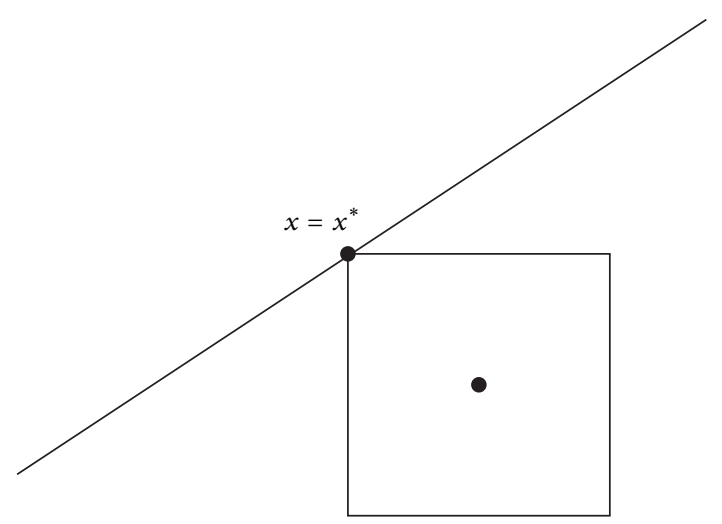

(b)

FIgURE 1: The minimizers to the mean square (a) and $l_{1}$ (b) approaches.

Require: Measurement matrix $\Phi$, measurement vector $u=\Phi x$, sparsity level $s$

(1) Initialize: As a first step, initialize $I=\emptyset$ and the residual $r=u$ repeat $s$ times:

(2) Identify: Select the largest coordinate $\lambda$ of $y=\boldsymbol{\Phi}^{*} r$ in absolute value. Break ties lexicographically.

(3) Update: Add the coordinate $\lambda$ to the index set: $I \leftarrow I \cup \lambda$ and update the residual according to:

$$
\widehat{x}=\arg \min _{z}\left\|u-\Phi_{\left.\right|_{I}} z\right\|_{2} ; \quad r=u-\Phi \widehat{x}
$$

(4) return: Index set $I \in 1, \ldots, d$

Algorithm 1: Orthogonal matching pursuit (OMP) pseudocode.

4.3. Compressive Sampling Matching Pursuit. Compressive Sampling Matching Pursuit (CoSaMP) implementation is given by Algorithm 2. As far as the CoSaMP algorithm is concerned, the sampling operator $\Phi$ is supposed to satisfy the restricted isometry property (for more information and a complete description of RIP please refer to [29]) and each $s$ coordinates of signal $y=\Phi^{*} \Phi x$, also called proxy signal, are close in terms of Euclidian norm to the $s$ corresponding coordinates of $x$.

The algorithm operates according to the following steps.

(1) Identification: the algorithm takes the residual as a proxy and locates its highest coordinates.

(2) Support merging: at the iteration $k$, the set of recently identified coordinates is merged with the set from iteration $k-1$.

(3) Estimation: based on the set of coordinates, the algorithm performs a least square to determine an approximation of the target signal.

(4) Pruning: in the estimated signal from least squares, the algorithm retains only the highest coordinates.

(5) Sample updating: the samples are updates so that they integrate the residual part.

4.4. Compressed Sensing for Spectrum Sensing and Primary Users Localization. In this paper, we will use the above framework in order to solve two major issues enabling CR: spectrum sensing and terminals localization. In order to do so, we will tend in our analysis to express the upcoming equations as the following:

$$
y=\boldsymbol{\Phi} x
$$

where $y \in \mathbb{R}^{M}$ is the measured entity, $\Phi \in \mathbb{R}^{M \times N}$ the measurement matrix, and $x \in \mathbb{R}^{N}$ the $K$-sparse vector to be reconstructed.

According to restricted isometry property definition, $\Phi$ would verify the RIP if $M \geq O(K \log (N / K))$.

\section{System Model}

In the considered system model, we will suppose that we do dispose of $N_{\mathrm{ch}}$ available channels in a wideband wireless network. Over a large geographic area, let $N_{p}$ be the number of deployed primary users using a different channel each. In this large area, we disperse $N_{c}$ cognitive radios that will cooperate to locate PUs in the network and detect their channels usage and states. The measures made by these cognitive terminals will then be sent to the fusion center. In order to enable SUs transmissions, the secondary network has to be aware of the availability and the state of each channel. Thus, SUs have to estimate which channels are occupied and to identify the PUs transmission powers and locations.

For our system, we adopt the path loss model, given by

$$
L(f, d)=P_{0}+20 \lg (f)+10 n \lg (d)[\mathrm{dB}],
$$

where $P_{0}$ is a constant related to antennas gain; $f$ is the channel frequency; $n$ is the path loss exponent; $d$ is the distance 
Require: Measurement matrix $\Phi$, measurement vector $u=\Phi x$, sparsity level $s$

(1) Initialize: Set $a^{0}=0, v=u, k=0$.

Repeat the following steps while increasing $k$ until achieving halt criterion.

(2) Signal Proxy: Set $y=\Phi^{*} v, \Omega=\operatorname{supp} y_{2 s}$ and merge the support $T=\Omega \bigcup \operatorname{supp} a^{k-1}$

(3) Signal Estimation: Solving a least squares problem, set: $b_{\left.\right|_{T}}=\Phi_{T^{\dagger}}^{\dagger} u$ and $b_{T_{T^{c}}}=0$

(4) Prune: Preparing the next iteration, set $a^{k}=b_{s}$

(5) Sample Update: Update the samples by: $v=u-\Phi a^{k}$

(6) return: $s$-sparse reconstructed vector $\widehat{x}=a$

Algorithm 2: Compressive sampling matching pursuit (CoSaMP) pseudocode.

separating the transmitting and receiving nodes; $\lg (\cdot)=$ $\log _{10}(\cdot)$.

In our case, we dispose of $N_{\text {ch }}$ channels, thus $f$ would be assumed the central frequency of each band, that is, $f \in$ $\left\{f_{0}, f_{1}, \ldots, f_{N_{\mathrm{ch}}-1}\right\}$.

Let us keep in mind that the path loss is related to the unknown channel and location of the PU. The received signal power is a combination of the unknown transmit power with the path loss expressed in (7).

Our task is to infer from the received signal at the cognitive terminals all this unknown, but valuable, information about the primary users.

\section{Compressed Sensing for Cognitive Radio Applications}

6.1. Spectrum Sensing. For discrete signals, the time domain samples $t$ are used to construct the spectrum in frequency domain using directly the DFT transform as follows:

$$
f=\mathbf{F} t
$$

where $\mathbf{F}$ is the normalized DFT matrix.

As stated previously, the sparsity in this context may come from the inability of the ADC to acquire signals at a Nyquist rate. The time samples $t$ are thus acquired at a sub-Nyquist rate which may result in a sparse vector.

We will thus directly apply the CS formalism with the different introduced algorithms to reconstruct the original time domain transmitted signal and spectrum sensing will be achieved using energy detection.

6.2. Location Estimation Based on Compressed Sensing. Once spectrum reconstructed and spectrum sensing achieved, more information can be derived while looking deeper into channels occupied by primary users.

Let us assume that in a certain wide area, PUs are located at coordinates $\left(x p_{m}, y p_{n}\right)$, where $x p_{m} \in\{0, \Delta x p, \ldots,(M-$ 1) $\Delta x p\}$ are $M$ possible $x$-axis positions (abscissæ) of the PUs (when we say $x p_{m} \in\{0, \Delta x p, \ldots,(M-1) \Delta x p\}$, that does not mean that there are $M$ PUs, but it means that $N_{p}$ primary users abscissæ (for ordinates as well) do actually have a finite "dictionary"); $y p_{m} \in\{0, \Delta y p, \ldots,(N-1) \Delta y p\}$ are $N$ possible $y$-axis positions (ordinates) of the PUs; $\Delta x p$ and $\Delta y p$ are, respectively, the resolutions over $x$-axis, and $y$-axis. Here, we do impose and suppose to the PU coordinates to be in discrete $M \times N$ dictionary (which, actually, is always true). It is good to remind at this level that the exact positions of the $N_{p}$ PUs $\left\{\left(x p_{i}, y p_{i}\right) ; i \in\left[1, \ldots, N_{p}\right]\right\}$ are unknown to our problem.

The $N_{c}$ CRs positions in the network are located at positions $\left\{\left(a_{i}, b_{i}\right) ; i \in\left[1, \ldots, N_{c}\right]\right\}$ (on which we do not impose being in a finite set, even if they necessarily are).

For the $k$ th $\mathrm{CR}$, sensing the $i$ th channel, the contribution of the PU located at the $\left(x p_{m}, y p_{n}\right)$ position on the received PSD is

$$
\begin{aligned}
& R_{k, i}(m, n)=P(m, n, i) \times 10^{L\left(f_{i}, d(m, n, k)\right) / 10}, \\
& d(m, n, k)=\sqrt{\left(x p_{m}-a_{k}\right)^{2}+\left(y p_{n}-b_{k}\right)^{2}}
\end{aligned}
$$

where $P(m, n, i)$ is the power transmitted by a PU using the $i$ th channel, located at $\left(x p_{m}, y p_{n}\right) ; f_{i}$ is the center frequency of the $i$ th channel; $d(m, n, k)$ represents the distance between the $k$ th CR and the the PU located at $\left(x p_{m}, y p_{n}\right)$.

The total received power over all the existing PUs, that is over the $M \times N$ possible positions of the PUs, can be formulated as the following:

$$
\begin{gathered}
Y_{k, i}=\sum_{m} \sum_{n} R_{k, i}(m, n), \\
Y_{k, i}=\sum_{m} \sum_{n} 10^{L\left(f_{i}, d(m, n, k)\right) / 10} \times P(m, n, i), \\
Y_{k, i}=L^{T}(k, i) P(i),
\end{gathered}
$$

where $P(i)$ is the vector containing the transmission power of the over all $M \times N$ grid over the $i$ th channel; $L(k, i)$ is the path loss vector computed according to (7) from all PU possible positions at the level of the $k$ th $\mathrm{CR}$, on the $i$ th channel. Consider

$$
\begin{gathered}
L(k, i)=10^{L_{\mathrm{dB}}(k, i) / 10} \\
L_{\mathrm{dB}}(k, i)=\left[L\left(f_{i}, d(0,0, k)\right), L\left(f_{i}, d(1,0, k)\right), \ldots,\right. \\
\left.L\left(f_{i}, d(m, n, k)\right), \ldots, L\left(f_{i}, d(M, N, k)\right)\right]^{T} .
\end{gathered}
$$

Let us denote by $Y_{k}=\left[Y_{k, 1}, \ldots, Y_{k, N_{\mathrm{ch}}}\right]^{T}$ the received signal power vector at the level of the $k$ th CR over the $N_{\text {ch }}$ available 
channels. This according to (10), and adopting the previous notation, can be expressed as

$$
Y_{k}=\mathbf{L}_{\mathbf{k}} P,
$$

where $P$ is the vector containing the transmission power of the $M \times N$ grid of PU locations over the $N_{c h}$ available channels of the $N_{C}$ deployed CRs as follows:

$$
P_{k}=\left[P^{T}\left(i_{1}\right), P^{T}\left(i_{2}\right), \ldots, P^{T}\left(i_{N_{C}}\right)\right]^{T} .
$$

The matrix $\mathbf{L}_{\mathbf{k}}$ is the fading gain matrix grouping at the level of the $k$ th CR the loss path contributions of the $M \times N \mathrm{PU}$ positions. The $j$ th row of $\mathbf{L}_{\mathbf{k}}$ is

$$
\mathbf{L}_{\mathbf{k}}(j)=\left[0,0, \ldots, L^{T}(k, j), 0, \ldots, 0\right] .
$$

Combining all the equations describing the $N_{C}$ CR system, we do obtain

$$
Y=\mathbf{L} P,
$$

where $Y=\left[Y_{1}^{T}, \ldots, Y_{N_{C}}{ }^{T}\right]^{T}$ and $\mathbf{L}=\left[\mathbf{L}_{1}, \ldots, \mathbf{L}_{\mathbf{N}_{\mathbf{C}}}\right]$.

The equation we ended with in (15) reminds us of (6) of the CS formalism we introduced previously: as $P$ is an unknown but sparse vector because over the $M \times N$ area we have been considering, only $N_{P}$ PUs are deployed in this area.

The two stages, spectrum sensing and localization, seem then to be attached to the same CS framework we have introduced before.

6.3. Joint Spectrum Sensing and Primary User Localization. We have shown till now that both problems sensing and localization can separately be solved using CS formalism. It is now easy for us to combine these two operations.

The joint framework would consider this dual sensing/localization problem as a $3 \mathrm{D}$ image reconstruction from sparse observation. The $x$ - axis and $y$-axis of this unknown image are the PU location information, the $z$-axis would represent the sensed channel occupancy information, and the value of a given pixel at $(x, y, z)$ is the reconstructed transmit power.

\section{Simulations and Results}

For our analysis, we suggest a realistic network simulation. In the considered CRS we deploy 5 PUs and 3 SUs. The 3 deployed CRs are attempting to communicate opportunistically and thus will perform the sensing and localization task.

A hexagonal cellular system functioning at $1.8 \mathrm{GHz}$ with a secondary cell of radius $R=1000 \mathrm{~m}$ and a primary protection area of radius $R_{p}=600 \mathrm{~m}$ is considered. Secondary transmitters may communicate with their respective receivers of distances $d<R_{p}$ from the BS. We assume that the PUs and the SUs are randomly distributed in a two-dimensional plane as shown in Figure 2 . The BS is placed at the center $(0,0)$. The distance, $d(m, n, k)$, from the $k$ th SU to the $\mathrm{PU}(m, n)$ is given by

$$
d(m, n, k)=\sqrt{\left(x p_{m}-a_{k}\right)^{2}+\left(y p_{n}-b_{k}\right)^{2}},
$$

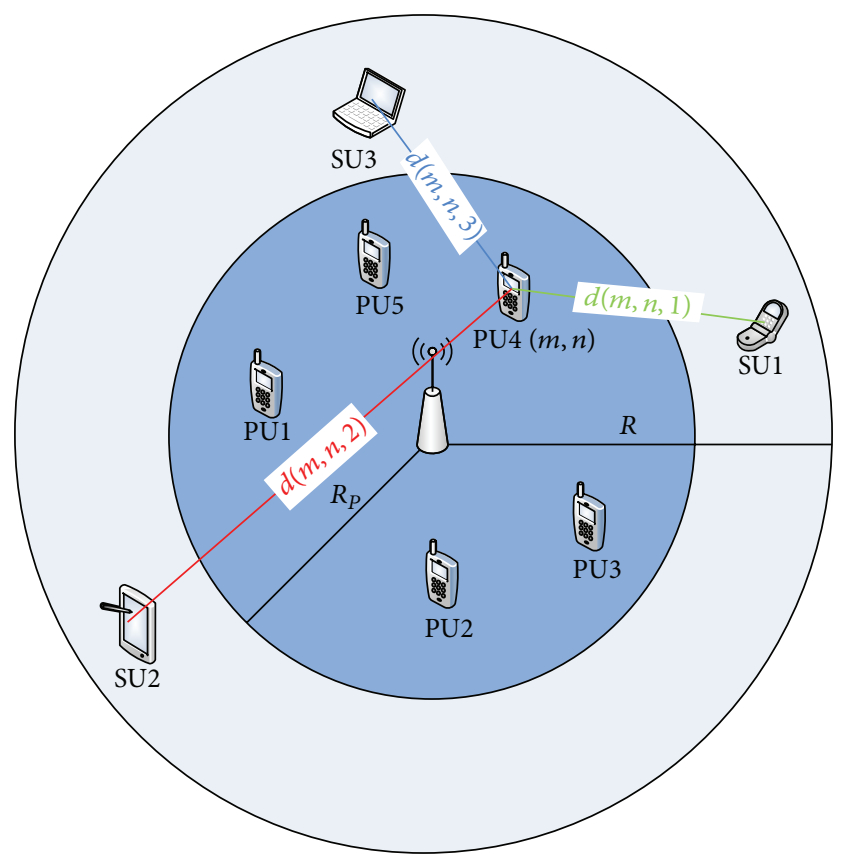

Figure 2: Two-dimensional plane of the cognitive radio system topology with five primary users and three secondary users.

where $\left(x_{p_{m}}, y_{p_{n}}\right)$ are the coordinates of the PU and $\left(a_{k}, b_{k}\right)$ the coordinates of the $k$ th CR. The channel gains are based on the COST-231 Hata model [30] including log-normal shadowing with standard deviation of $10 \mathrm{~dB}$, plus fast-fading assumed to be i.i.d. circularly symmetric with distribution $\mathscr{C} \mathcal{N}(0,1)$. The basic path loss for the COST-231 Hata model which is in $\mathrm{dB}$ in an urban area at a distance $d$ is

$$
\begin{aligned}
L(f, d)= & 46.3+33.9 \log _{10}\left(f_{c}\right)-13.82 \log _{10}\left(h_{b}\right) \\
& -A_{M}+\left(44.9-6.55 \log _{10}\left(h_{b}\right)\right) \log _{10}(d)+C_{M},
\end{aligned}
$$

where $f_{c}$ is the carrier frequency equal to $1.5 \mathrm{GHz}$ and $h_{b}$ is the base antenna height equal to 50 meters. The distance $d$ is computed using (16). $C_{M}$ is $0 \mathrm{~dB}$ for medium-sized cities and suburbs and is $3 \mathrm{~dB}$ for metropolitan areas. In the simulations, we use $C_{M}=0 \mathrm{~dB}$. The $A_{M}$ is defined for urban environment as

$$
A_{M}=3.20\left(\log _{10}\left(11.75 h_{m}\right)\right)^{2}-4.97,
$$

where $h_{m}$ is the mobile antenna height equal to 10 meters. The shadowing variations of the path loss can be calculated from the log-normal distribution as follows:

$$
g(x \mid \sigma)=\frac{1}{\sigma \sqrt{2 \pi}} \exp \left(\frac{-x^{2}}{2 \sigma^{2}}\right)
$$

where $\sigma$ is the variability of the signal equal to $10 \mathrm{~dB}$. The shadowing variation is computed using the Matlab function randn. Shadowing reflects the differences in the measured received signal power with relation to the theoretical value calculated by path loss formulas. Averaging over many 
received signal power values for the same distance however yields the exact value given by path loss.

Furthermore, for channels distribution, we suppose that the total number of available channels is in $[1,2, \ldots, 20]$ channels and each of the five PUs is communicating over a single different channel.

For simulations, we generate a random sequence with 5 of the 20 channels (a single channel per user is assumed), where the amplitude (PSD) is also randomly generated per user. This generated spectrum is then fixed once and for all in the simulations. For spectrum sensing, in order to model the ADC imperfections, we generate a random $M \times N$ matrix as stated in $[3,6]$, and the sparse signal is then the generated signal passed through the random matrix plus a white noise at a given SNR. After this generation of the sparse noisy signal, we process using the several reconstruction algorithms and compute the spectrum reconstruction error. For location estimation, distance is derived using the CS proposed formalism with pathloss expression given in (17). Unlike spectrum generation, here, noise is implicitly inherited from the received noisy signal.

7.1. Simulation Results. Figures 3 and 4 give an example of spectrum reconstruction MSE (we recall the definition of mean square error as MSE $=\|X-\widehat{X}\|^{2}$, where $X$ is the original observation and $\widehat{X}$ is the reconstructed signal) at 50\% sparsity for the simulated algorithms and the impact of sparsity level on spectrum reconstruction MSE.

The very first remark that we can give here is that the simulation results are in line with the theoretical aspects related to BP, OMP, and CoSaMP. As we explained at the very beginning, performance related to $\mathrm{BP}$ is expected to overcome OMP, which is the case in Figure 3, and CoSaMP is shown both in theory and simulations to outperform OMP, but it is still not as efficient as BP.

Figure 4 is assessing the impact of sparsity level on spectrum reconstruction MSE. Here we clearly see that results are coherent with the definition we gave of sparsity, saying that "a $d$-dimensional vector is assumed to be $s$-sparse if it has $s$ or fewer nonzero coordinates." According to this definition, the more sparse the vector is, the larger its support is, so the less the reconstruction MSE would be, which is perfectly in line with the results reported in Figure 4.

Figures 5 and 6 give an example of PU location estimation at $50 \%$ sparsity for the simulated algorithms and the impact of sparsity level on PU location estimation error at $0 \mathrm{~dB}$.

Figure 5 gives an overview of PU location estimation error at 50\% sparsity level for BP, OMP, and CoSaMP. Here we validate again the previous results, as the tendency of $\mathrm{BP}$ outperforming CoSaMP and OMP is confirmed. The same for Figure 6, where we clearly see the more signal sparse is (in the sense the more we have nonzero entries) the more robust the PU localization is.

Assuming a 15 meters limit of "good localization" bound, Figure 5 shows that the idea of cooperative localization could be exploited up to $-3 \mathrm{~dB},-2 \mathrm{~dB}$, and $1 \mathrm{~dB}$ for $\mathrm{BP}$, CoSaMP, and OMP, respectively. These performances are obtained for totally autonomous GPS free techniques and with absolutely no need for extra overhead data exchange in the network.

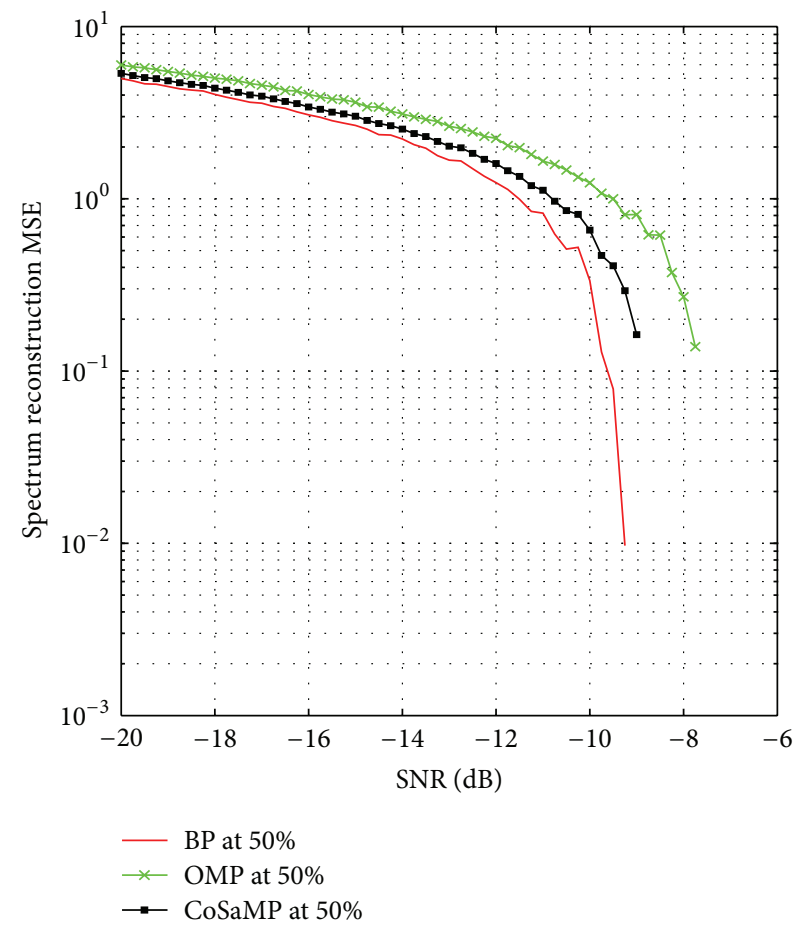

FIGURE 3: Example of spectrum reconstruction MSE at 50\% sparsity level for BP, OMP, and CoSaMP.

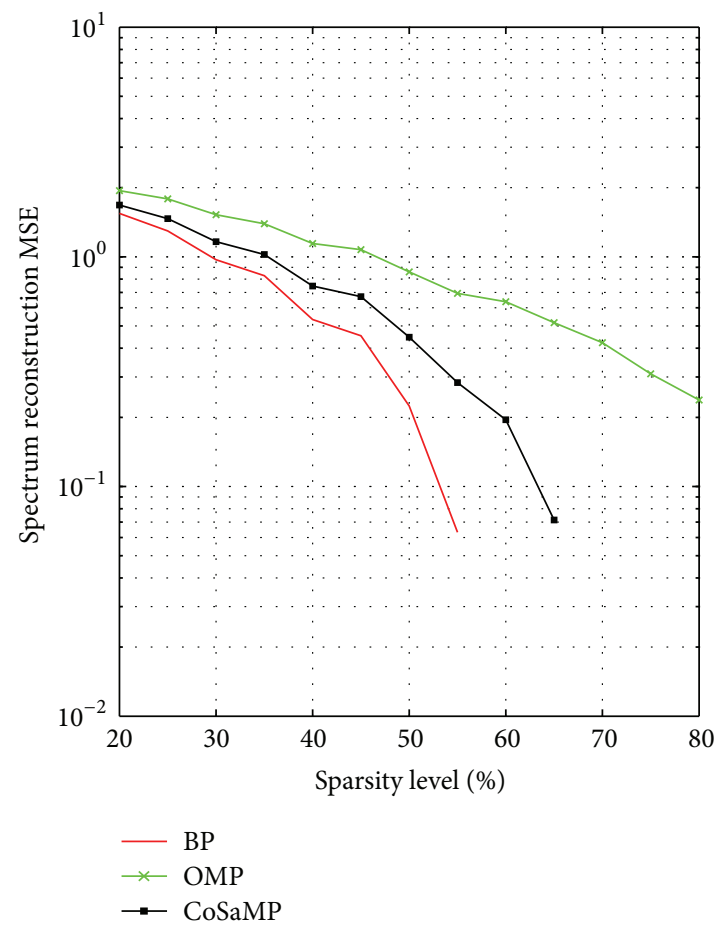

FIGURE 4: Impact of sparsity on spectrum reconstruction MSE at $0 \mathrm{~dB}$. 


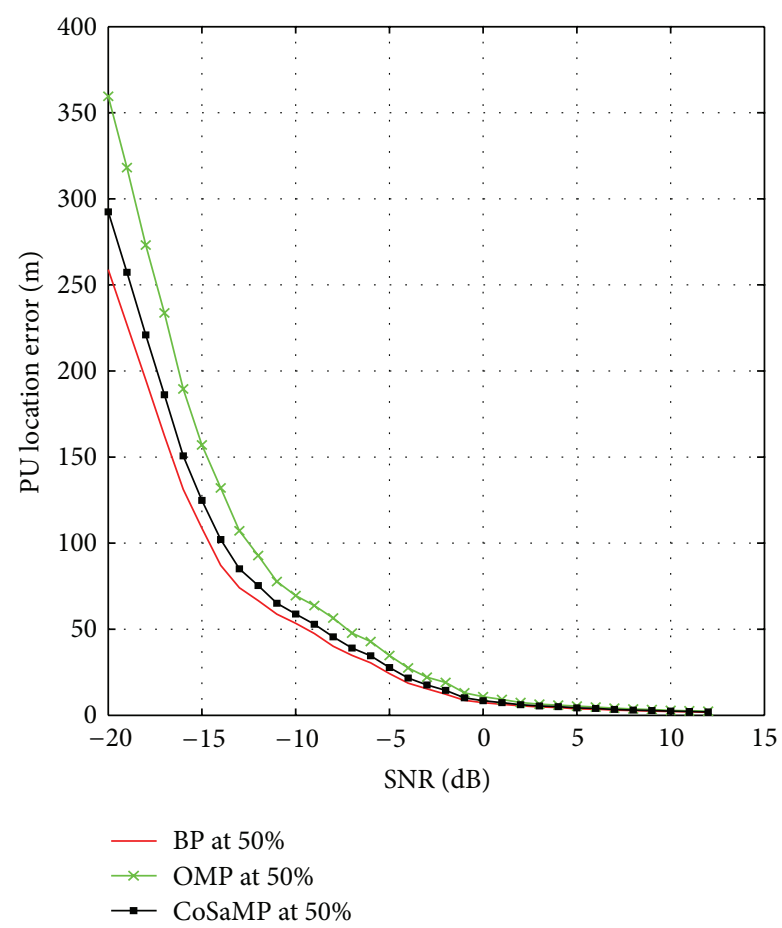

Figure 5: Error on PU location estimation at 50\% sparsity level for $\mathrm{BP}, \mathrm{OMP}$, and CoSaMP.

\section{Conclusion}

This work presents a first look towards a combined spectrum sensing and localization task. These two tasks are fundamental in order to enable cognition in wireless networks. With the combination of the two tasks, we also considered a realistic data acquisition constraint, which is sparsity due to the ADC technology limits. Simulation results of the proposed technique show promising and interesting results for compressed sensing techniques applied to this formalism.

The formalism that we derived here is the starting point of a whole location-aided cognitive radio framework that is being developed within the FP7 WHERE2 project. In this framework, once the radio map [31] is built thanks to the sensing and localization tasks, the CRS are thus able to communicate in the available bands in the available directions and thus the proposed framework is a key enabler for space division multiple access (SDMA) systems and spatial versions of CR paradigms (underlay, overlay, and interweave). This formalism can also be exploited for D2D (device to device) communication, as the CRs can communicate directly with each other while a fusion center can play the role of the control entity in the network.

\section{Acknowledgments}

EURECOM's research is partially supported by its industrial members: ORANGE, BMW Group, Swisscom, SFR, ST Microelectronics, Symantec, SAP, Monaco Telecom, and iABG and also by the EU FP7 projects WHERE2 and

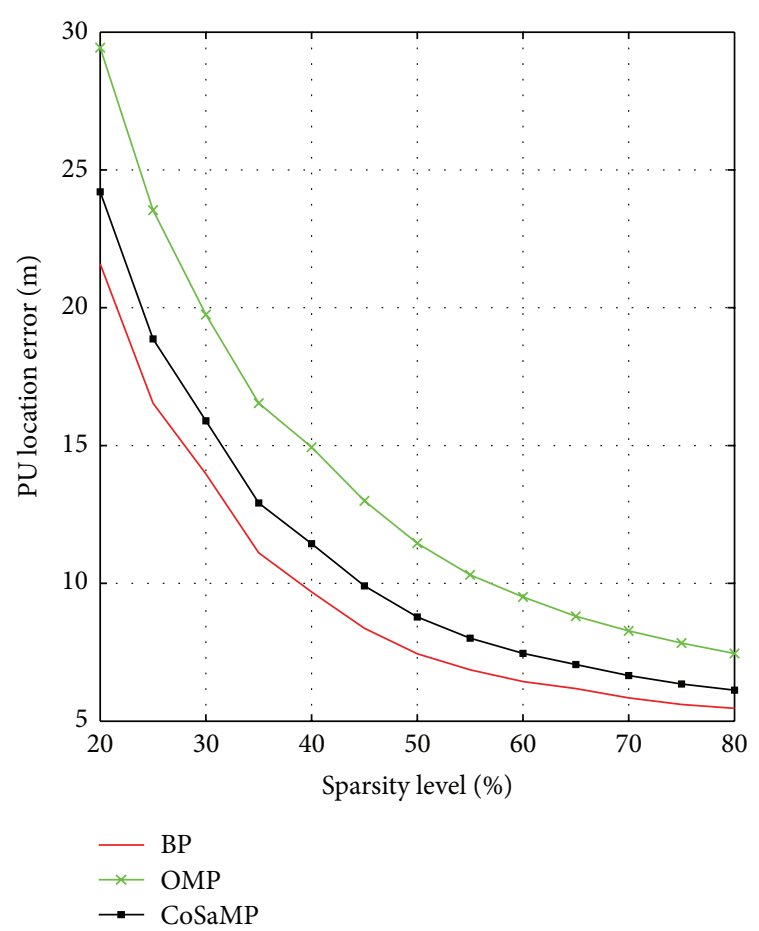

FIgURE 6: Impact of sparsity on PU location estimation at $0 \mathrm{~dB}$.

NEWCOM\#. The authors would like to thank the reviewers for their constructive comments.

\section{References}

[1] S. Haykin, "Cognitive radio: brain-empowered wireless communications," IEEE Journal on Selected Areas in Communications, vol. 23, no. 2, pp. 201-220, 2005.

[2] J. Mitola III and G. Q. Maguire Jr., "Cognitive radio: making software radios more personal," IEEE Personal Communications, vol. 6, no. 4, pp. 13-18, 1999.

[3] E. J. Candes, "Compressive sampling," in Proceedings of the International Congress of Mathematicians, Invited Lecture, Madrid, Spain, August 2006.

[4] Z. Tian and G. B. Giannakis, "Compressed sensing for wideband cognitive radios," in Proceedings of the IEEE International Conference on Acoustics, Speech and Signal Processing (ICASSP '07), pp. IV1357-IV1360, April 2007.

[5] M. A. Davenport, M. B. Wakin, and R. G. Baraniuk, "Detection and estimation with compressive measurements," Tech. Rep., Department of ECE, Rice University, 2006.

[6] E. J. Candès, J. K. Romberg, and T. Tao, "Stable signal recovery from incomplete and inaccurate measurements," Communications on Pure and Applied Mathematics, vol. 59, no. 8, pp. 12071223, 2006.

[7] E. J. Candes and T. Tao, "Decoding by linear programming," IEEE Transactions on Information Theory, vol. 51, no. 12, pp. 4203-4215, 2005.

[8] E. J. Candes and T. Tao, "Near-optimal signal recovery from random projections: universal encoding strategies?" IEEE Transactions on Information Theory, vol. 52, no. 12, pp. 5406-5425, 2006.

[9] E. J. Candes and M. B. Wakin, "An introduction to compressive sampling: a sensing/sampling paradigm that goes against the 
common knowledge in data acquisition," IEEE Signal Processing Magazine, vol. 25, no. 2, pp. 21-30, 2008.

[10] W. Guibene and D. Slock, "A combined spectrum sensing and terminals localization technique for cognitive radio networks," in Proceedings of the IEEE 8th International Conference on Wireless and Mobile Computing, Networking and Comm's (WiMob '12), 2012.

[11] W. Guibene and D. Slock, "A compressive sampling approach for spectrum sensing and terminals localization in cognitive radio networks," in Proceedings of the IEEE 17th International Workshop on Computer Aided Modeling and Design of Communication Links and Networks (CAMAD '12), Barcelona, Spain, October 2012.

[12] M. G. Khoshkholgh, K. Navaie, and H. Yanikomeroglu, "Access strategies for spectrum sharing in fading environment: overlay, underlay, and mixed," IEEE Transactions on Mobile Computing, vol. 9, no. 12, pp. 1780-1793, 2010.

[13] G. Ding, Q. Wu, F. Song, X. Li, and J. Wang, "Joint exploration and exploitation of spatial-temporal spectrum hole for cognitive vehicle radios," in Proceedings of the IEEE International Conference on Signal Processing, Communications and Computing (ICSPCC '11), September 2011.

[14] S. Mumtaz, J. Bastos, J. Rodriguez, and C. Verikoukis, "Adaptive beamforming for OFDMA using positioning information," in Proceedings of the Wireless Advanced (WiAd '11), pp. 7-14, June 2011.

[15] WHERE2 Consortium, "Location-aided PHY/MAC Layer Design for Advanced Cognitive Radios," Deliverable D3.3, 2012, http://www.kn-s.dlr.de/where2/index.php.

[16] P. Cheraghi, Y. Ma, and R. Tafazolli, "Frequency-domain differential energy detection based on extreme statistics For OFDM source sensing," in Proceedings of the IEEE 73rd Vehicular Technology Conference (VTC '11-Spring), May 2011.

[17] W. Guibene and D. Slock, "Degrees of freedom of downlink single- and multi-cell multi-user MIMO systems with location based CSIT," in Proceedings of the IEEE 77th Vehicular Technology Conference (VTC '13-Spring), Dresden, Germany, 2013.

[18] I. F. Akyildiz, W.-Y. Lee, M. C. Vuran, and S. Mohanty, "NeXt generation/dynamic spectrum access/cognitive radio wireless networks: a survey," Computer Networks, vol. 50, no. 13, pp. 2127-2159, 2006.

[19] T. Yücek and H. Arslan, "A survey of spectrum sensing algorithms for cognitive radio applications," IEEE Communications Surveys and Tutorials, vol. 11, no. 1, pp. 116-130, 2009.

[20] S. Xu, Z. Zhao, and J. Shang, "Spectrum sensing based on cyclostationarity," in Proceedings of the IEEE Workshop on Power Electronics and Intelligent Transportation System (PEITS '08), pp. 171-174, August 2008.

[21] B. Zayen, Spectrum sensing and resource allocation strategies for cognitive radio [Ph.D. thesis], Telecom PariTech, 2010.

[22] S. Muthukrishnan, Data Streams: Algorithms and Applications, Now Publishers, 2005.

[23] J. A. Tropp and A. C. Gilbert, "Signal recovery from random measurements via orthogonal matching pursuit," IEEE Transactions on Information Theory, vol. 53, no. 12, pp. 4655-4666, 2007.

[24] D. L. Donoho, Y. Tsaig, I. Drori, and J.-L. Starck, "Sparse solution of underdetermined systems of linear equations by stagewise orthogonal matching pursuit," IEEE Transactions on Information Theory, vol. 58, no. 2, pp. 1094-1121, 2012.
[25] M. Fornasier and H. Rauhut, "Iterative thresholding algorithms," Applied and Computational Harmonic Analysis, vol. 25, no. 2, pp. 187-208, 2008.

[26] T. Blumensath and M. E. Davies, "Iterative hard thresholding for compressed sensing," Applied and Computational Harmonic Analysis, vol. 27, no. 3, pp. 265-274, 2009.

[27] D. Needell and R. Vershynin, "Uniform uncertainty principle and signal recovery via regularized orthogonal matching pursuit," Foundations of Computational Mathematics, vol. 9, no. 3, pp. 317-334, 2009.

[28] D. Needell and J. A. Tropp, "CoSaMP: iterative signal recovery from incomplete and inaccurate samples," Applied and Computational Harmonic Analysis, vol. 26, no. 3, pp. 301-321, 2009.

[29] E. J. Candès, “The restricted isometry property and its implications for compressed sensing," Comptes Rendus Mathematique, vol. 346, no. 9-10, pp. 589-592, 2008.

[30] COST 231, "Urban transmission loss models for mobile radio in the 900- and $1800 \mathrm{MHz}$ bands," TD(90)119 Revision 2, September, 1991.

[31] Y. Zhao, Enabling cognitive radios through radio environment maps [Ph.D. thesis], Virginia Polytechnic Institute and State University, 2007. 

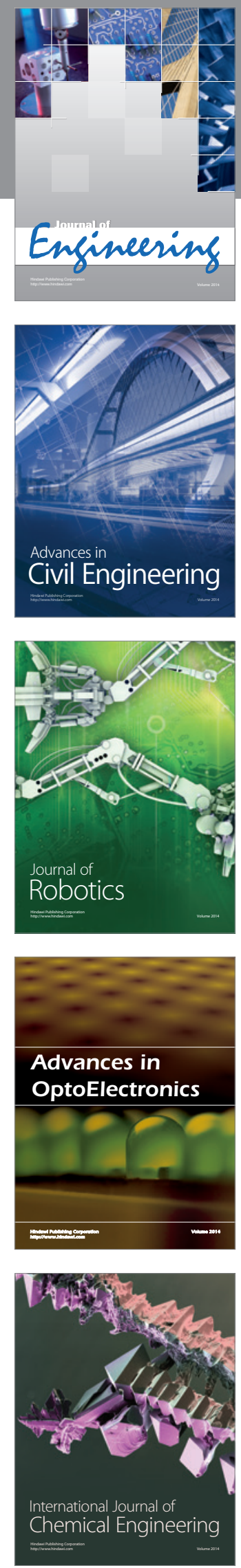

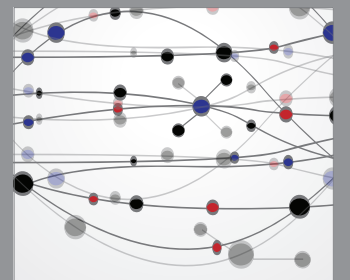

The Scientific World Journal
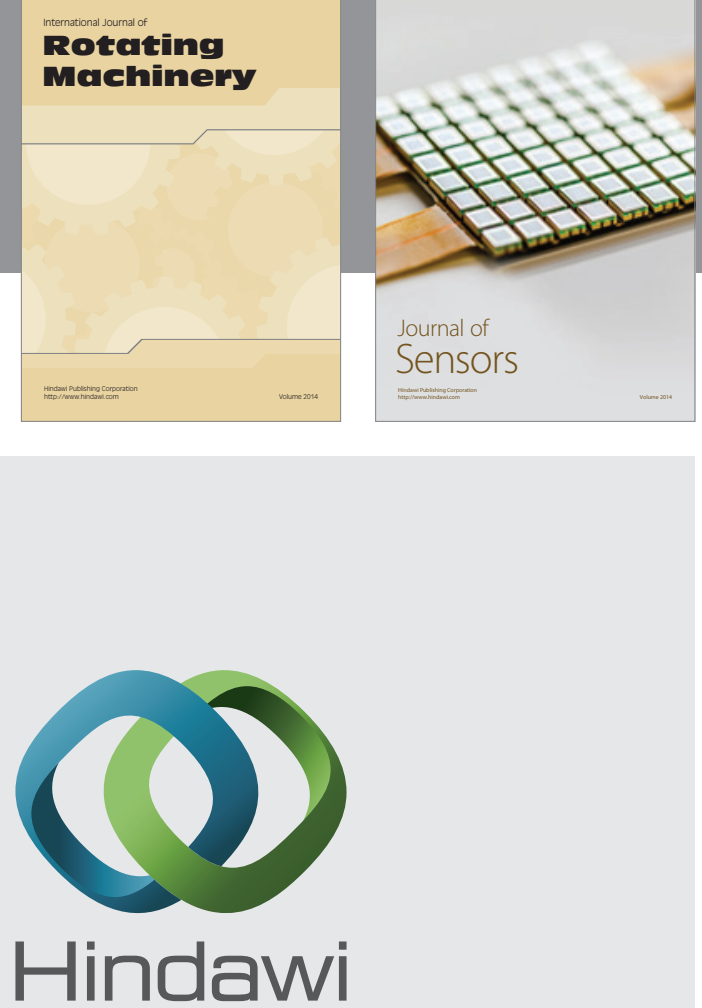

Submit your manuscripts at http://www.hindawi.com
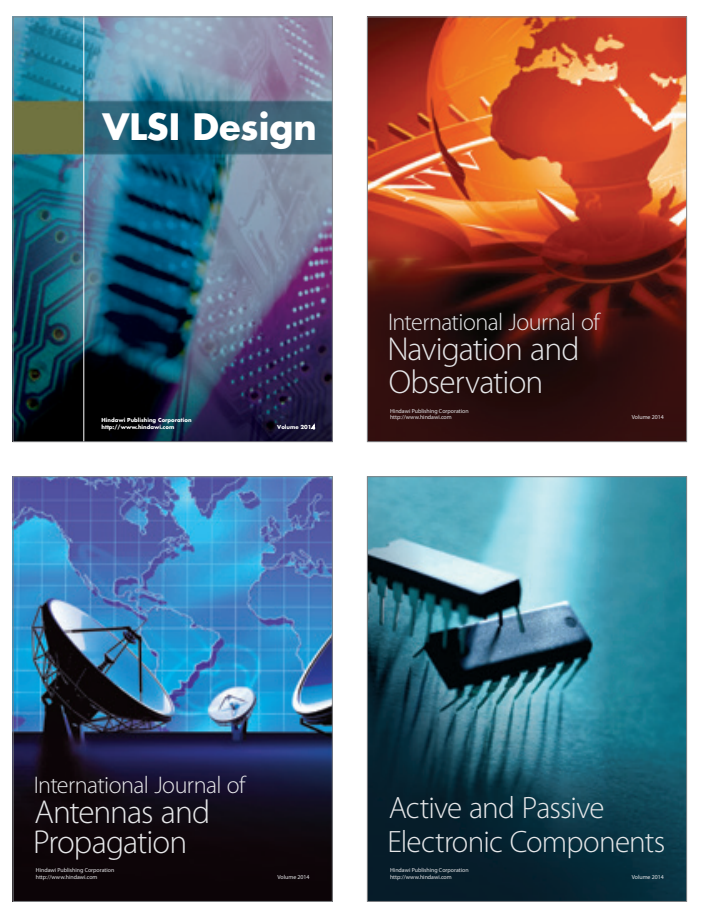
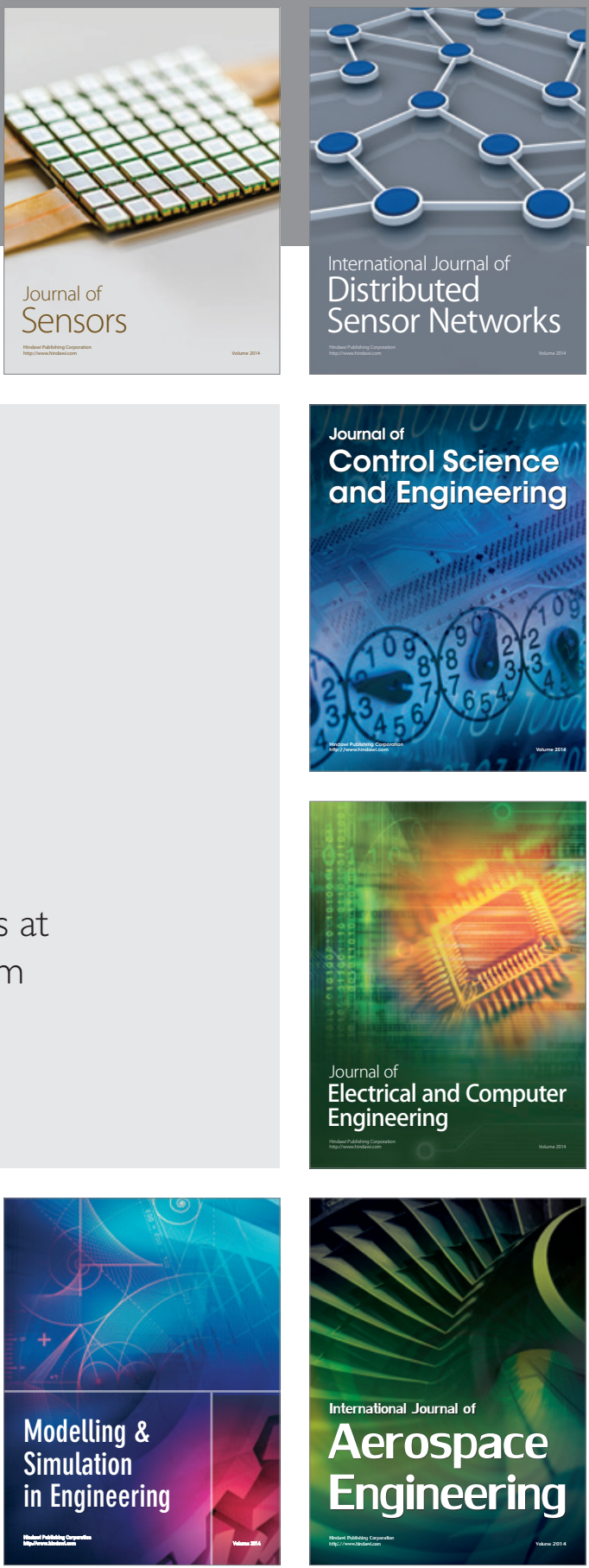

Journal of

Control Science

and Engineering
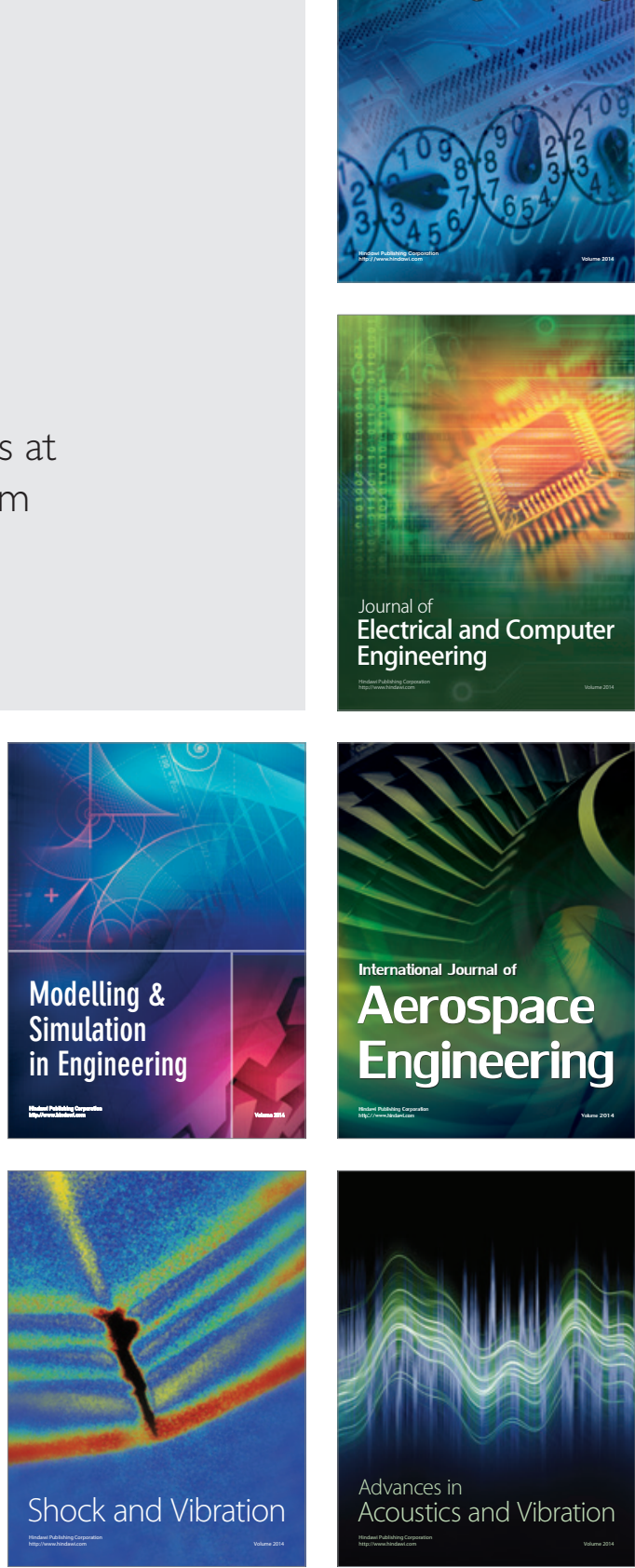